\title{
¿Cómo realizar un mapeo de temperatura en un área de almacenamiento de medicamentos?
}

\section{How to conduct a temperature mapping in a drug storage area?}

\section{Pág. 10-13}

Fabricio Montes Campos, Estudiante de Farmacia en la Universidad de Ciencias Médicas (UCIMED),San José, Costa Rica Adriana Bolaños Carpio, Regente del Instituto de Investigación en Ciencias Médicas (ICIMED), San José, Costa Rica.

Recibido: 18/11/19

Aprobado: 20/11/19

\section{Resumen:}

Las droguerías en Costa Rica deben cumplir con el Reglamento de Buenas Prácticas de Almacenamiento y Distribución de Medicamentos en Droguerías. Uno de los puntos clave de este reglamento es garantizar que la calidad de los medicamentos se mantenga y la mejor forma de mantenerla es controlando las condiciones ambientales durante su almacenamiento. La temperatura es uno de los parámetros que más afecta la estabilidad de los medicamentos y por ello se deben realizar mapeos de temperatura para establecer en cuáles áreas es seguro almacenar los productos. En este artículo se detallan los pasos que un farmacéutico necesita saber para realizar de forma exitosa un mapeo de temperatura.

Abstract: Drugstores in Costa Rica must comply with the Regulation of Good Practices for Storage and Distribution of Drug Products. One of the key points of this regulation is to verify that the quality of drug products is maintained and the best way to maintain it is to control the environmental conditions during storage. Temperature is one of the parameters that most affects the stability of drug products and therefore temperature mapping should be performed to establish in which areas is safe to store drug products. In this article we intend to detail the steps that a pharmacist needs to know to successfully perform a temperature mapping.

Palabras claves: mapeo de temperatura, temperatura cinética media, almacenamiento de medicamentos.

Keywords: Temperature Mapping, Mean Kinetic Temperature, Drug Storage.

\section{Introducción}

En la actualidad, y desde el año 2013, toda droguería en Costa Rica debe cumplir con el Decreto $\mathrm{N}^{\circ}$ 37700-S, titulado Reglamento de Buenas Prácticas de Almacenamiento y Distribución de Medicamentos en Droguerías. Uno de los puntos clave de este reglamento es garantizar que la calidad de los medicamentos se mantenga y se contribuya así con la salud de la población (4).

Partiendo del hecho de que la droguería cuenta con áreas limpias, secas y seguras, la mejor forma de mantener la calidad de los medicamentos es controlando las condiciones ambientales durante su almacenamiento.

La temperatura es el mayor influyente en las áreas de almacenamiento, debido a que puede llegar a alterar el producto y dañarlo (2). No mantener el rango de temperatura establecido en el etiquetado del producto puede causar la ausencia o el cambio 
de su acción terapéutica y provocar efectos adversos en la salud del paciente (3). Para evitar esto, se realiza lo que se conoce como un mapeo de temperatura.

En este artículo se detallan los pasos que un regente de una Droguería necesita saber para realizar de forma exitosa un mapeo de temperatura.

\section{¿Qué es un mapeo de temperatura?}

El mapeo de temperatura es el proceso de recolección de datos de temperatura en diferentes zonas del área de almacenamiento de la droguería.

La importancia de este proceso es que permite determinar si las zonas donde se almacenan los medicamentos cumplen con los rangos establecidos en la etiqueta del producto.

Para la realización del mapeo, se usa el concepto de Temperatura Cinética Media (MKT, por sus siglas en inglés) que es un parámetro aceptado internacionalmente para definir las condiciones de temperatura en los estudios de estabilidad a largo plazo y difiere del promedio aritmético (2). La MKT es una forma simple de explicar el efecto total de los cambios de temperatura durante el tiempo de almacenamiento de los productos sensibles al calor. La Farmacopea de los EE.UU. (USP 38) la define como: "Una temperatura de almacenamiento isotérmica que simula los efectos no isotérmicos de la variación de la temperatura de almacenamiento" (6).

La MKT permite observar el estrés térmico adquirido en el tiempo por un producto cuando ha sido almacenado y distribuido, el cual ha sufrido cambios de temperatura en el tiempo (1).

Se requiere del procedimiento de mapeo de temperatura para todo espacio asignado al almacenamiento y manipulación de productos con una temperatura de almacenamiento etiquetada especificada. Esto incluye salas de congelador, cámaras frigoríficas, áreas de almacenamiento con temperatura controlada, áreas de cuarentena y bahías de recepción y carga. También puede incluir laboratorios. Es importante mencionar que los rangos de temperatura permitidos en estas áreas variarán (1).

\section{¿Cómo se realiza un mapeo de temperatura?}

La Organización Mundial de la Salud propone las siguientes etapas para la realización de este proceso. En su reporte técnico número 961 se detallan a cabalidad los pasos seguir, sin embargo, aquí los hemos resumido (5):

\section{Etapa 1.Preparación de un protocolo}

Antes de ejecutar el mapeo, primero se debe establecer el protocolo. De esta manera se definen los pasos respectivos y se disminuyen los factores de error.

El protocolo debe contener ciertos puntos específicos, como por ejemplo descripción, alcance, objetivos, entre otros; sin embargo, el de mayor interés es el de la metodología, que se debe usar como guía para la ejecución del mapeo y debe incluir que el estudio debe realizarse por un período de siete días consecutivos, en las diferentes estaciones del año como lo indica nuestra legislación (4).

Para la operación del mapeo de temperatura, se requiere de varios sensores de temperatura (data loggers), dependiendo del tamaño del área de almacenamiento. En espacios pequeños, de menos de $20 \mathrm{~m} 2$, se usan como mínimo 4 data loggers. Estos aparatos deben tener un amplio rango de temperatura para poder medir temperaturas extremas, por ejemplo, de $-30^{\circ} \mathrm{C}$ hasta $+60^{\circ} \mathrm{C}$, deben permitir intervalos de medición

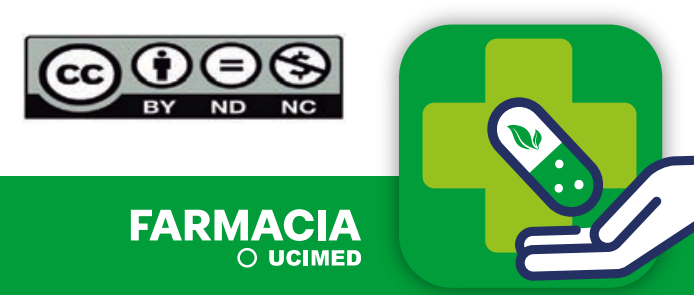


de tiempo, por ejemplo, desde 1 minuto hasta 15 minutos máximo, y deben contar con memoria suficiente para guardar dichas repeticiones (4).

\section{Etapa 2. Ejecución}

Para la ejecución del mapeo se deben seguir los siguientes pasos:

1.Seleccionar los sensores de temperatura a usar. Deben estar calibrados.

2.Designar el personal que realizará el mapeo de temperatura.

3.Evaluar las áreas que se analizarán.

4.Establecer los criterios de aceptación. Por ejemplo, que se considere aceptable temperaturas entre 15 y $30{ }^{\circ} \mathrm{C}$ y a un rango de humedad relativa del 30 al $70 \%$, según el producto a almacenar.

5.Determinar la localización de los data logger.

6.Registrar los data loggers y los sitios donde se colocan.

7.Etiquetar y programar los data loggers.

8.Ejecutar el mapeo: dejar que los sensores registren la temperatura y la humedad relativa cada 15 minutos durante 7 días consecutivos.

9.Analizar los datos recolectados.

\section{Etapa 3. Elaboración del reporte}

Este reporte debe incluir los datos recolectados, analizados y las conclusiones del mapeo de temperatura según los estándares previamente establecidos, donde se pueden identificar desvíos o problemas relacionados y las recomendaciones para solventarlos.

\section{Etapa 4. Implementación de las recomendaciones.}

Una vez identificados los hallazgos en la etapa anterior, se procede a resolverlos mediante acciones correctivas tan sencillas como incluir un deshumedecedor o más complejas como cambiar todo el sistema de ventilación, calefacción 0 de aire acondicionado. Es recomendable que se ejecute un seguimiento a las acciones correctivas para valorar su eficacia.

Finalizadas estas 4 etapas, el mapeo está listo y se estará cumpliendo con lo requerido en el Decreto $N^{\circ} 37700-S$ que también indica que este proceso debe realizarse cada tres años o cada vez que se realicen modificaciones estructurales en las áreas de almacenamiento que puedan afectar la temperatura de los medicamentos.

\section{Conclusión}

El llevar un control de la temperatura y humedad en una droguería o farmacia es imprescindible debido a que cada producto tiene sus propias condiciones de almacenamiento que si se desvían puede dañarse y provocar consecuencias no deseadas al paciente que lo usa. A su vez, podría resultar en un problema económico que el establecimiento farmacéutico afrontaría, ya que tendría pérdidas de material, producto y además tiempo de producción.

Asimismo, es importante realizar un mapeo de temperatura y de humedad en las áreas para identificar si los equipos utilizados para el mantenimiento de la temperatura y humedad están en correcto funcionamiento, de lo contrario se debe buscar el medio para repararlo o sustituirlo (2).

El estudio de mapeo térmico también sirve para evaluar la infraestructura de la droguería, el sistema de climatización o ventilación, los factores externos ambientales, y hasta los procesos logísticos del almacén. 


\section{Bibliografía}

[1]. Flórez O, Rojas J, Gallardo C. Análisis de la temperatura propuesta por la Conferencia Internacional sobre Armonización (ICH) para Colombia en los estudios de estabilidad natural de medicamentos. VITAE [Internet]. 2005 [cited 23 September 2019]; (12):56-61. Available from: http://www.scielo.org.co/ pdf/vitae/v12n1/v12n1a07.pdf

[2]. Gallardo C, Rojas J, Flórez O. La Temperatura Cinética Media en los estudios de estabilidad a largo plazo y almacenamiento de los medicamentos. VITAE [Internet]. 2004 [cited 23 September 2019]; (11):68. Available from: https://www.redalyc.org/pdf/1698/169818259009.pdf

[3]. Pyatigorskaya NV, Vasilyevich V, Viktorovich V, Vladimirovich S, Mikhaylovich A. Rationale for the Necessity of Temperature Mapping of Storage Areas for Pharmaceutical Products. Journal of Pharmaceutical Sciences and Research [Internet]. 2018 [cited 23 September 2019]; (10): 662-664. Available from: https://www.jpsr.pharmainfo.in/Documents/Volumes/vol10lssue03/jpsr10031850.pdf

[4]. Reglamento de Buenas Prácticas de Almacenamiento y Distribución de Medicamentos en Droguerías. Decreto N 37700-S. 28/11/2013. http://www.pgrweb.go.cr/scij/Busqueda/Normativa/Normas/ nrm_texto_completo.aspx?param1=NRTC\&nValor1=1\&nValor2=74926\&nValor3=92670\&strTipM=TC. (Fecha de consulta: 23 de setiembre del 2019.)

[5]. Temperature mapping of storage areas. Technical supplement to WHO Technical Report Series, No. 961, 2011 Annex 9: Model guidance for the storage and transport of time and temperature-sensitive pharmaceutical products. 2014. pp 8-15. https://www.who.int/biologicals/expert_committee/Supplement-8-TS-mapping-storage-areas-ECSPP-ECBS.pdf (Fecha de consulta: 23 de setiembre del 2019.) [6]. United States Pharmacopeia 38. $<1079>$ Good storage and distribution practices for drug products. p. 1134 